\title{
GLAZAR-2: A WIDE-FIELD CAMERA ABOARD THE MIR SPACE STATION
}

\author{
H.M. TOVMASSIAN ${ }^{1}$, Y.M. KHODJAYANTS ${ }^{2}$, M.N. KRMOYAN ${ }^{2}$, R.V. \\ ISADJANIAN $^{2}$, O.N. GASPARIAN ${ }^{2}$, A.Z. ZAKHARIAN ${ }^{2}$ and D. HUGUENIN ${ }^{3}$ \\ ${ }^{1}$ Byurakan Astrophysical Observatory, Armenia, and \\ INAOE, Tonantzintla, Mexico \\ 2 'Granit' Special Construction Bureau, Armenia \\ ${ }^{3}$ Geneva Observatory, Switzerland
}

In 1987 the UV imaging telescope Glazar (Tovmassian et al. 1988) was launched to the Mir Space Station. Its sensitivity was lower than was expected. For this reason the UV survey of the sky was not done and only observations of OB stellar associations were made. The fields of about 15 stellar associations and of the Large Magellanic Cloud were observed. Only images of O, B or early A type stars were obtained on the Glazar photographs at $1640 \AA$. The observations allowed the detection of an average $\sim 15 \%$ new, unknown OB type stars in each of the observed fields, the discovery of hot unknown components of some late type stars and a detailed study of the distribution of stars in space. Some new, unknown OB stellar associations were discovered and more correct distances of stellar associations were determined. The distribution of the absorbing matter was also studied. New, B-type stellar associations, named B were discovered.

Glazar-2 is a wide field camera for obtaining prime images at $1670 \AA$. It basically has the same parameters as Glazar, but has definite important advantages. First, due to absence of correcting lenses it is by about $4^{\mathrm{m}}$ more sensitive. Second, due to larger limits of rotations around both axes $\left( \pm 35^{\circ}\right)$ and faster movements it is possible to make observations at the orbital orientation of the Space Station, while in the case of the Glazar the observations could be done only at inertial orientation of the whole Space Station, when the latter was pointed during observations toward the observed field on the sky. Third, in the case of the Glazar-2 the photo camera is replaceable through the same air lock through which cassettes with exposed films are changed.

Like Glazar it was designed and manufactured at the 'Granit' Special Construction Bureau in Armenia. The parameters of Glazar-2 are as follows:

Optical system:

Diameter of the main mirror:

Focal length:

Field of view:

Angular resolution;

Limiting stellar magnitude at $1670 \AA$ :

Detector: modified Ritchey-Chretien.

$40 \mathrm{~cm}$.

$1.7 \mathrm{~m}$.

$1.3(40 \mathrm{~mm})$.

$\sim 10$ arcsec.

$\sim 17^{\mathrm{m}} .5$.

micro channel intensifier + film .

The telescope functions at the orbital orientation of the Mir Space Station. It has two star 55 
trackers: rough and fine. First the one, with a field-of-view of $4^{\circ}$ 'catches' the guiding offset star after the entrance of the Space Station in the shadow part of the orbit. After 'bringing' the star to the centre of the field-of-view the second fine star tracker operates. Its field-of-view is 30 arcmin. The accuracy of guidance is about 2.5 arcsec. The star trackers work with stars up to 5-th stellar magnitude in $B$.

The star trackers move in relation to the optical axis of the telescope along a line to a parallel position, shifted by 0.9 . Thus two neighbouring photographed fields are partly overlapped. Due to precession, the position angle of this line changes and in principle a whole field of about $5^{\circ}$ in diameter around each guiding star could be observed.

The telescope has two modes of operation. In the first case, the coordinates of the field to be observed and the exposure time are introduced to the control system of the telescope by cosmonauts. In the second case, the same is introduced from the ground by telemetry. The telescope may observe only one field or can observe at all parallel positions of the star trackers, automatically moving them. Also by command it may make in a sequence several photographs with different exposures.

The cassettes with exposed films are changed through the special air lock. Through the same air lock the whole photo camera (detector, power supply and cassette) may be changed.

The telescope was launched to the Mir Space Station in 1990. The checking after the launch showed that all devices of the telescope were functioning well. But after the first experimental try to change the cassette, the handle of the air lock was broken. Later it was fixed. But during this time the photo camera was left not in a proper position and it is now out of use. But it is possible to replace it and to use a new one. The replacement could have a digital output.

\section{References}

Tovmassian, H.M., Khodjayants, Yu.M., Krmoyan, M.N., Kashin, A.L., Zakharian, A.Z., Hovhannessian, R.Kh., Mkrtchian, M.A., Tovmassian, G.H., Huguenin, D., Bootov, V.V., Romanenko, Yu.V., Laveikin, A.I. and Alexandrov, A.P., 1988. Pis'ma v Astron. Zh., 14, 291. 